\title{
Effets De La Variabilite Climatique Sur Le Rendement De Quelques Cultures Vivrieres Dans Le Nord-Est Du Benin
}

\author{
Hounzinme Sènadé Sylvie \\ Docteur en Climatologie Appliquée \\ Unité de Recherche en Phytosociologie, Espaces Protégés et Pastoraux, \\ Agro-écosystèmes, Conservation et valorisation des espèces endogènes \\ Laboratoire de Recherche en Biologie Appliquée (LARBA) \\ Ecole Polytechnique d'Abomey- Calavi (EPAC) \\ Chanhoun Comlan Silvère Landry, \\ Faculté des Sciences Agronomiques, Université d'Abomey-Calavi \\ Teka Oscar, \\ Maître de Conférences à la Faculté des Sciences Agronomiques (FSA) \\ de l'Université d'Abomey-Calavi (UAC), \\ Laboratoire d'Ecologie Appliquée (LEA)

\section{Oumorou Madjidou,} \\ Professeur Titulaire à l'Université d'Abomey-Calavi (UAC) \\ Unité de Recherche en Phytosociologie, Espaces Protégés et Pastoraux, \\ Agro-écosystèmes, Conservation et valorisation des espèces endogènes \\ Laboratoire de Recherche en Biologie Appliquée (LARBA) \\ Ecole Polytechnique d'Abomey- Calavi (EPAC)
}

Doi:10.19044/esj.2020.v16n12p137 URL:http://dx.doi.org/10.19044/esj.2020.v16n12p137

\section{Résumé}

Plusieurs études effectuées ces dernières années au Bénin révèlent que l'agriculture est de plus en plus affectée par les effets néfastes de la variabilité climatique. La présente étude vise à déterminer les effets de la variabilité climatique sur les rendements agricoles des spéculations telles que le maïs, le niébé, le sorgho, et l'arachide dans le Nord-est du Bénin et d'évaluer la conformité entre les données climatiques et la perception des producteurs de la variabilité climatique. La méthodologie adoptée est l'analyse de régression linéaire entre les anomalies de températures, de pluviométrie et de rendements préalablement calculés pour les quatre cultures et par Commune d'étude sous le logiciel R 3.3.1, suivie des entretiens structurés sur la perception des producteurs face à la variabilité climatique et le rendement agricole, effectués auprès de 240 producteurs répartis dans quatre Communes du milieu d'étude. 
Une AFC et un test Chi2 ont été effectués sur les données de perception. Ainsi, de l'analyse des modèles linéaires simples réalisés entre les anomalies de rendements et les anomalies de paramètres agro climatiques, il ressort une significativité globale des variables de la température et de la pluviométrie sur le rendement du maïs, du sorgho, du niébé et de l'arachide. Les paramètres de températures ( $\mathrm{T}$ max, Tmoy, Tmin) et de précipitations qui influent sur les rendements de cultures varient en fonction du milieu et de la spéculation agricole. De plus, en se référant à la perception des producteurs de l'effet de la variabilité climatique sur la production agricole, l'inondation et la mauvaise répartition des pluies sont les manifestations observées à Kandi et Nikki tandis qu'à Bembèrèkè et Tchaourou, c'est plutôt la rareté des pluies et les vents violents. Ces manifestations ont pour causes, selon les enquêtés, la déforestation $(90 \%)$, les phénomènes naturels $(57,92 \%)$, le non-respect des interdits $(42,92 \%)$, avec pour conséquences sur les cultures, la baisse des rendements de maïs, sorgho, niébé et arachide.

Mots clés : Variabilité climatique, Rendement agricole, Modèle linéaire simple, Nord-Est Bénin 


\title{
Effects of Climate Variability on Agricultural Yields of Some Food Crops in the North-East of Benin
}

\author{
Hounzinme Sènadé Sylvie \\ Docteur en Climatologie Appliquée \\ Unité de Recherche en Phytosociologie, Espaces Protégés et Pastoraux, \\ Agro-écosystèmes, Conservation et valorisation des espèces endogènes \\ Laboratoire de Recherche en Biologie Appliquée (LARBA) \\ Ecole Polytechnique d'Abomey- Calavi (EPAC) \\ Chanhoun Comlan Silvère Landry, \\ Faculté des Sciences Agronomiques, Université d'Abomey-Calavi \\ Teka Oscar, \\ Maître de Conférences à la Faculté des Sciences Agronomiques (FSA) \\ de l'Université d'Abomey-Calavi (UAC), \\ Laboratoire d'Ecologie Appliquée (LEA)

\section{Oumorou Madjidou,} \\ Professeur Titulaire à l'Université d'Abomey-Calavi (UAC) \\ Unité de Recherche en Phytosociologie, Espaces Protégés et Pastoraux, \\ Agro-écosystèmes, Conservation et valorisation des espèces endogènes \\ Laboratoire de Recherche en Biologie Appliquée (LARBA) \\ Ecole Polytechnique d'Abomey- Calavi (EPAC)
}

\begin{abstract}
Different studies show that these last years in Benin the agriculture is affected harshly by the climatic variability. The present work aims at determining the effects of climate variability on agricultural yields of foodcrops, such as maize, cowpea, sorghum, and groundnut in the North-east of Benin and to assessing the compliance between the climate data and the perception of farmers regarding climate variability. For this purpose two types of data were used. There were agricultural statistics of crop yields and meteorological data. In addition structured interviews were conducted with 240 farmers in the four districts of the study area. Questions of the interviews were related to the perception of the farmer of climate variability and effects on agricultural production. Data processing consisted of the calculation of anomalies as well a annual crops yields indexes for each of the four (04) targeted crops. A regression analysis had been carried out using crops yields and meteorological parameters for the period 1981 to 2012. The analysis was performed in the R software 3.3.1 for regression analysis moreover AFC and
\end{abstract}


Chi2 test for perception data. Results, showed that the linear models using the anomalies of crop yields and the anomalies of climate parameter revealed a high link between both parameters. It stands out an over significance of the variables of temperature and rainfall on the yield of maize, cowpea, sorghum, and groundnut. In fact, the meteorological data (T max, Tmoy, Tmin, Pmoy) influence yields of foodcrops varied regardind districts and crops. Farmers perceive climate variability and their perceptions were the flooding, rain irregularity observed in Kandi and Nikki while in Bembèrèkè and Tchaourou are the rarity of the rains and the strong winds. These events are caused, according to the respondents, deforestation (90\%), natural phenomena (57.92\%), non-compliance with prohibitions (42.92\%), with consequences for crops, the decline in maize, cowpea, sorghum, and groundnut yields.

Keywords : Climate variability, Crop yield, linear model, North-east Benin

\section{Introduction}

La plupart des écosystèmes des régions agro écologiques sont aujourd'hui marqués par une dégradation du fait de la forte variabilité climatique associée à une plus grande fréquence des phénomènes extrêmes (sécheresse, augmentation de la température, etc.) au cours des trois dernières décennies (Ogouwalé 2006). L'Afrique est cité comme le continent le plus vulnérable aux changements climatiques (Ouedraogo, 2010). Ainsi, depuis la fin des années 60, les impacts de la variabilité climatique ont de plus été accentués en Afrique de l'Ouest, par une succession de décennies déficitaires de pluie (Amoussou et al., 2012). L'augmentation des températures, l'irrégularité et la mauvaise répartition des pluies ainsi que les événements exceptionnels tels que l'inondation et la sécheresse sont autant de facteurs qui compromettent sérieusement la sécurité des moyens de subsistance des personnes pauvres (Chede, 2012). Le continent africain est donc soumis à un climat fortement variable et imprévisible, ce qui fragilise les systèmes agricoles qui ne répondent plus aux pressions actuelles du climat (Yegbèmey et al., 2014). Cette forte variabilité aussi bien temporelle, spatiale que quantitative des précipitations rend les systèmes de production agricole plus vulnérables et constitue une contrainte majeure aux objectifs d'autosuffisance alimentaire que se sont fixés les pays de l'Afrique de l'Ouest (Agbossou, 2010). La variabilité climatique affecte la production de plusieurs façons par les incertitudes avec le début de la saison agricole, les inondations et la sécheresse dans certaines zones (Ikheloa et al., 2013). L'évolution des conditions climatiques a de graves répercussions négatives sur la production agricoles, la sécurité alimentaire et les ressources naturelles (Agbongiarhuoyi et al., 2013). L'agriculture joue un rôle crucial dans l'économie de la plupart des pays africains. Selon les estimations de la Banque Africaine de 
Développement (BAD) en 2010, $70 \%$ de la population dépend de l'agriculture pour l'emploi à plein temps, et de nombreux africains comptent sur l'agriculture pour une partie du revenu de leur ménage. Dans ces conditions où l'économie de la majorité des pays africains repose sur l'agriculture, les populations rurales de l'Afrique sub-saharienne sont particulièrement exposées aux aléas climatiques dans la mesure où elles sont étroitement dépendantes de l'agriculture pluviale qui représente près de $93 \%$ des terres cultivées (Sultan, 2011). De plus, l'accroissement rapide des populations (plus $3 \%$ par an entre 1990 et 2002), conjugué à la diminution des ressources alimentaires, renforce la vulnérabilité des populations africaines en raison de leur faible niveau de mécanismes d'adaptation (Odjugo, 2010).

Au Bénin, l'agriculture est pluviale et saisonnière, donc exposée aux effets de la variabilité climatique. Elle emploie près de $70 \%$ des actifs et contribue pour 32,6\% du PIB (AGVSAN, 2009). L'agriculture constitue donc la principale source d'alimentation des populations béninoises, mais reste vulnérable aux changements climatiques qui agissent sur l'ensemble du secteur (Teka et al., 2013). Toutes les régions du Bénin sont touchées par ces effets en particulier la zone du Nord où les poches de sécheresse en saison pluvieuse, l'arrêt des pluies, la variabilité spatio-temporelle, la modification du régime pluviométrique, la mauvaise répartition des pluies et les vents violents viennent respectivement en tête des risques climatiques (Hounkponou et al., 2011). L'agriculture vivrière, est sérieusement menacée par les effets pervers des changements climatiques (Honkpehedji et Agbo, 2009). Les producteurs agricoles sont les plus vulnérables aux effets néfastes de la variabilité climatique (Hounzinme et al., 2017) ; ils perçoivent les risques climatiques et développent donc des stratégies d'adaptations. Comment les paramètres climatiques (pluviométrie moyenne, les températures moyennes, minimales et maximales) affectent le rendement du maïs, du sorgho, du niébé et de l'arachide au Nord-est au Bénin ? Comment les producteurs agricoles perçoivent-ils les perturbations et leurs effets sur le rendement agricole?

Le présent travail a pour objectif de déterminer les effets de la variabilité climatique sur le rendement agricole des spéculations (maïs, sorgho, niébé et arachide) dans le Nord-est du Bénin et d'évaluer la perception des producteurs agricoles à la variabilité climatique.

\section{Matériel Et Méthodes}

\subsection{Milieu D'étude}

Le milieu d'étude englobe quatre (04) Communes du nord-est du Bénin. Il s'agit des Communes de Tchaourou, de Nikki, de Bembèrèkè et de Kandi. Ces Communes ont été choisies dans les départements du Borgou et de l'Alibori parce qu'elles font partie des grandes Communes productrices 
agricoles (produits vivriers) dans cette région septentrionale du pays. Elles sont caractérisées par un climat tropical avec une saison sèche de novembre à avril et une saison des pluies de juin à septembre. La hauteur annuelle moyenne de pluie est de $1100 \mathrm{~mm}$ et la température est comprise entre $24^{\circ} \mathrm{C}$ et $34^{\circ} \mathrm{C}$ (Issa, 2012). Les sols rencontrés au niveau de la zone d'étude sont de type ferrugineux tropical. Ce sont des sols ayant une profondeur plus ou moins importante. Leur perméabilité et leur porosité sont généralement bonnes. La figure 1 présente la localisation des Communes où les travaux de terrain ont été conduits.

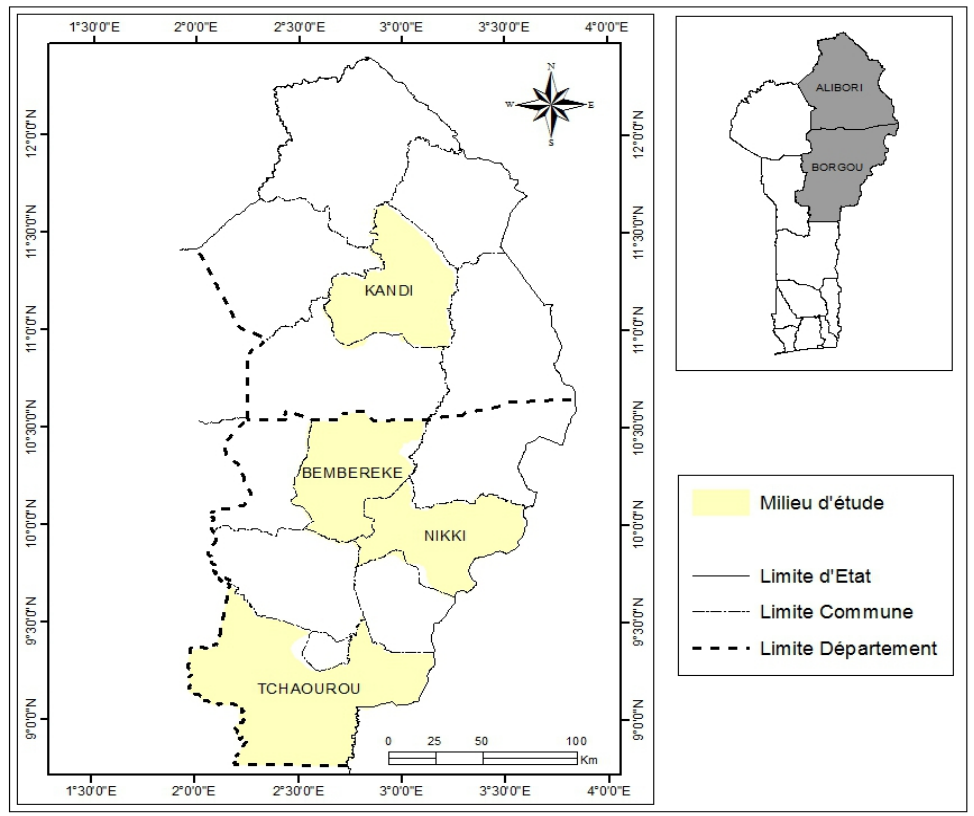

Figure 1 : Localisation du milieu d'étude

\section{2. Méthodes de collecte des données}

\subsubsection{Evaluation des effets des paramètres climatiques sur le rendement}

Pour étudier les effets de la variabilité climatique sur le rendement, deux types de données ont été utilisés dans la présente étude. Il s'agit des données climatiques (température minimale, maximale et la pluviométrie) et des statistiques de rendements agricoles annuels. Les données climatiques (1981 à 2012) recueillies au niveau de la Météo Bénin ont été complétées par des informations des stations synoptiques de Kandi et de Parakou. Quant aux statistiques agricoles, l'Agence Territoriale de Développement Agricole (ATDA) Borgou/Alibori est la structure de référence par le biais des ex SCDA (Service Communal de Développement Agricole) des Communes de Tchaourou, Bembèrèkè, Nikki et Kandi.A cet effet, plusieurs documents ont été consultés dont les annuaires statistiques agricoles de 1981 à 2012, les 
rapports des compagnes agricoles et les rapports d'activités de 1981 à 2012 . Dans cette étude, quatre (04) spéculations ont été retenues. Il s'agit du maïs (Zea mays), du sorgho (Shorgum bicolor), du niébé (Vigna unguiculata) et de l'arachide (Arachis hypogaea). L'évolution des rendements agricoles de ces quatre (04) spéculations dans chacune des quatre Communes de la zone d'étude a été appréciée de 1981 à 2012.

\subsubsection{Perception paysanne de la variabilité climatique}

Pour l'étude de la perception des producteurs agricoles de la variabilité, la méthodologie utilisée est une combinaison d'approches de collectes de données à la fois quantitatives et qualitatives. Les informations ont été collectées auprès des producteurs agricoles de la zone d'étude. La taille de l'échantillon a été déterminée à partir de la formule de Dagnelie (1998):

$$
n=\frac{P_{i}\left(1-P_{i}\right) t_{1-\alpha / 2}^{2}}{d^{2}}
$$

Pi $(88,75 \%)$ obtenu à partir d'une enquête exploratoire réalisée dans la zone d'étude sur 80 producteurs. Elle représente la proportion des producteurs qui cultivent au moins trois (03) des quatre spéculations (maïs, niébé, arachide et sorgho). $\mathrm{t} 1-\alpha / 2=1,96$, valeur de la variable aléatoire normale pour un risque $\alpha$ de 0,05 . La marge d'erreur d prévue pour tout l'échantillonnage est de 4\%. La taille $\mathrm{n}$ de l'échantillon est ainsi sensiblement égale à 240 producteurs. En absence de données par rapport à la proportion des producteurs enquêtés dans chaque Commune, le nombre de producteurs interrogés a été répartie à part égale, soit 60 par Commune.

\subsection{Traitement des données}

\subsubsection{Analyse des effets des paramètres climatiques sur le rendement des cultures}

Les statistiques agricoles ont été épurées et traitées dans le tableur Excel. Ensuite, les anomalies de rendements annuels pour chacune des quatre (04) spéculations ont été calculées ainsi que les anomalies de précipitations et de températures (minimales, maximales, moyennes). La formule de l'indice de Lamb des anomalies, définie comme une variable centrée réduite, a permis le calcul des anomalies (Ardoin-Bardin, 2004). On l'exprime par l'équation:

$$
\boldsymbol{I}_{\boldsymbol{P}}=\left(\boldsymbol{X}_{\boldsymbol{i}}-\overline{\boldsymbol{X}}\right) / \boldsymbol{\sigma}
$$

Où $X_{i}, \bar{X}$ et $\sigma$ désignent respectivement les valeurs des paramètres climatiques annuels étudiés ou le rendement annuel de l'année i; la moyenne interannuelle de ces paramètres ou du rendement des cultures sur la période considérée (1981 à 2012) et l'écart-type de la série.

Une analyse de régression a été réalisée afin d'apprécier l'impact de la variabilité climatique sur les rendements agricoles du maïs, sorgho, niébé et 
arachide dans les quatre Communes. Elle a été possible grâce aux anomalies de températures, de précipitation et de rendements des quatre spéculations agricoles étudiées. L’analyse a été effectuée dans le logiciel R3.3.1 (R CoreTeam, 2016).

\subsubsection{Analyse de la perception paysanne}

L'Analyse factorielle des correspondances (AFC) a été réalisée pour décrire la perception des différents producteurs agricoles sur les manifestations de la variabilité climatique. Cette analyse a été réalisée grâce au logiciel R. Le test de $\mathrm{Chi}^{2}$ a été effectué pour tester la dépendance de la perception des causes de la variabilité climatique avec les différentes Communes.

\section{Résultats}

\subsection{Effets des paramètres climatiques sur le rendement agricole}

\section{Commune de Tchaourou}

Le lien entre le rendement et les paramètres climatiques s'est révélé significatif $(\mathrm{P}<0,05)$ selon les spéculations de l'étude à Tchaourou entre les années 1981 et 2010 (Tableau 1). En effet, les températures minimales et moyennes expliquent de façon significative le rendement de l'arachide. Le rendement de l'arachide est positivement corrélé avec la température minimale et moyenne. Les températures minimales allant de 21,93 à $22,4^{\circ} \mathrm{C}$ et celles moyennes comprises entre 27,31 et $28,6^{\circ} \mathrm{C}$ donnent les meilleurs rendements. De la même manière, les températures minimales et la pluviométrie moyenne ont eu un effet significatif $(\mathrm{P}<0,05)$ sur le rendement du maïs. Ainsi, les faibles températures associées à une pluviométrie moyenne améliorent le rendement de maïs. Les meilleurs rendements du maïs sont obtenus pour les valeurs de température minimale comprise entre 21,95 et $22,4^{\circ} \mathrm{C}$; et une pluviométrie moyenne comprise entre 1177,20 et $1490,78 \mathrm{~mm}$. Il en est de même pour le sorgho, positivement corrélé avec la température minimale, moyenne et la pluviométrie moyenne. Ces rendements sont meilleurs lorsque la température minimale est comprise entre 21,93 et $22,4^{\circ} \mathrm{C}$; celle moyenne entre 27,31 et $28,6^{\circ} \mathrm{C}$ et la pluviométrie moyenne comprise entre 1136 et $1490 \mathrm{~mm}$. Par ailleurs, le rendement du niébé est significativement influencé par la température, présentant ainsi une corrélation positive de façon globale.

Tableau 1. Equation de régression entre le rendement des spéculations et les paramètres climatiques dans la Commune de Tchaourou

\begin{tabular}{|l|l|l|l|l|}
\hline Spéculations & T min & Tmax & Tmoy & Pmoy \\
\hline Maïs & $\begin{array}{l}\text { AR= }-0,43+0,6 \\
\text { An Tmin; } p=0,009\end{array}$ & NS & NS & $\begin{array}{l}\text { AR= -0,08+0,32An } \\
\text { Pmoy } ;=0.02\end{array}$ \\
\hline Sorgho & $\begin{array}{l}\text { AR=-0,57+0,9 An } \\
\text { Tmin; } p=0,0001\end{array}$ & NS & $\begin{array}{l}\text { AR=0,30+1,10 } \\
\text { An Tmoy ; } \\
p=0,002 /\end{array}$ & $\begin{array}{l}\text { AR=-0,01+0,33 An } \\
\text { Pmoy ; } p=0,04\end{array}$ \\
\hline
\end{tabular}




\begin{tabular}{|c|c|c|c|c|}
\hline Spéculations & $T$ min & Tmax & Tmoy & Pmoy \\
\hline Niébé & $\begin{array}{l}\mathrm{AR}=-0,63+1,03 \\
\mathrm{An} \quad \mathrm{Tmin} ; \\
\mathrm{p}=0,0000186\end{array}$ & $\begin{array}{l}\mathrm{AR}=- \\
0,23+0, \\
44 \quad \mathrm{An} \\
\mathrm{Tmax} ; \\
\mathrm{p}=0,01\end{array}$ & $\begin{array}{l}\mathrm{AR}=- \\
0,31+1,25 \mathrm{An} \\
\text { Tmoy } \\
\mathrm{p}=0,0002\end{array}$ & NS \\
\hline Arachide & $\begin{array}{l}\mathrm{AR}=-0,51+0,77 \\
\text { An Tmin; } \mathrm{p}=0,001\end{array}$ & NS & $\begin{array}{lr}\text { AR }= & 0,20+0,93 \\
\text { An } & \text { Tmoy; } \\
p=0,006 & \end{array}$ & NS \\
\hline
\end{tabular}

NS= Non Significatif

\section{$>$ Commune de Nikki}

L'Analyse des données de la Commune de Nikki montre les relations significatives entre le rendement et les températures minimales, maximales et moyennes de 1981 à 2010 (Tableau 2). De l'analyse du tableau, il ressort que les températures minimales et moyennes ont eu un effet significatif $(\mathrm{P}<0,05)$ sur le rendement de l'arachide. En effet, les meilleurs rendements de l'arachide ont été obtenus pour des valeurs de température minimale comprise entre $21,91-22,4^{\circ} \mathrm{C}$ et une température moyenne comprise entre 27,9 et $28,6^{\circ} \mathrm{C}$. De même, les températures de façon globale ont eu un effet significatif $(\mathrm{P}<0,05)$ sur le rendement du maïs dans la Commune de Nikki de 1981 à 2010. Donc, une corrélation positive existe entre le rendement du maïs et la température. Les meilleurs rendements du maïs ont été obtenus pour les valeurs de température minimale comprise entre 21,89 et $22,4^{\circ} \mathrm{C}$, une température moyenne comprise entre 27,27 et $28,6{ }^{\circ} \mathrm{C}$ et une température maximale comprise entre 33,46 et $34,9^{\circ} \mathrm{C}$. Enfin, s'agissant du sorgho, les températures minimale, moyenne et maximale ont eu un effet significatif sur le rendement. Le rendement du sorgho est positivement corrélé avec la température de façon globale. Ces rendements sont meilleurs pour des valeurs de températures minimales $\left(21,89\right.$ et $\left.22,4^{\circ} \mathrm{C}\right)$, températures moyennes $\left(27\right.$ et $\left.28,6^{\circ} \mathrm{C}\right)$ et les températures maximales $\left(33,46\right.$ et $\left.34,9^{\circ} \mathrm{C}\right)$.

Tableau 2. Equation de régression entre le rendement des spéculations et les paramètres climatiques dans la Commune de Nikki

\begin{tabular}{|c|c|c|c|}
\hline Spéculations & T min & Tmax & Tmoy \\
\hline Maïs & $\begin{array}{c}\mathrm{AR}=-0,33+0,58 \mathrm{An} \\
\text { Tmin; } \mathrm{p}=0,03\end{array}$ & $\begin{array}{c}\mathrm{AR}=-2,21+0,51 \mathrm{An} \\
\mathrm{Tmax} ; \mathrm{p}=0,008\end{array}$ & $\begin{array}{c}\mathrm{AR}=0,30+1,06 \text { An_Tmoy } ; \\
\mathrm{p}=0,003\end{array}$ \\
\hline Sorgho & $\begin{array}{l}\mathrm{AR}=-0,37+0,64 \mathrm{An} \\
\mathrm{Tmin} ; \mathrm{p}=0,018\end{array}$ & $\begin{array}{l}\mathrm{AR}=-0,25+0,59 \mathrm{An} \\
\mathrm{Tmax} ; \mathrm{p}=0,001\end{array}$ & $\begin{array}{c}\mathrm{AR}=0,35+1,21 \text { An Tmoy } \\
\mathrm{p}=0,0006\end{array}$ \\
\hline Niébé & $\begin{array}{l}\mathrm{AR}=-0,51+0,85 \mathrm{An} \\
\text { Tmin; } \mathrm{p}=0,001\end{array}$ & $\begin{array}{c}\mathrm{AR}=-0,22+048 \mathrm{An} \\
\mathrm{Tmax} ; \mathrm{p}=0,01\end{array}$ & $\begin{array}{c}\mathrm{AR}=0,32+1,719 \text { An Tmoy } ; \\
\mathrm{p}=0,0006\end{array}$ \\
\hline Arachide & $\begin{array}{l}\mathrm{AR}=-0,41+0,67 \mathrm{An} \\
\text { Tmin; } \mathrm{p}=0,01\end{array}$ & $\mathrm{NS}$ & $\begin{array}{c}\mathrm{AR}=0,21+0,82 \text { An Tmoy } \\
\mathrm{p}=0,0006\end{array}$ \\
\hline
\end{tabular}


Commune de Bembèrèkè

Dans cette Commune, plusieurs relations significatives ont été établies entre le rendement et les paramètres climatiques excepté la pluviométrie moyenne entre les années 1981 et 2010 (tableau 3). Les températures minimales et les températures moyennes influencent significativement $(\mathrm{P}<0,05)$ le rendement de l'arachide. Le rendement de l'arachide est positivement corrélé avec la température moyenne et minimale. Les températures minimales comprises entre 21,93 et $22,4^{\circ} \mathrm{C}$ et celles moyennes comprises entre 27,30 et $28,6^{\circ} \mathrm{C}$ donnent les meilleurs rendements. De même, les températures de façon globale améliorent significativement le rendement du niébé. Les meilleurs rendements du niébé ont été obtenus pour les valeurs de température minimale comprise entre 21,90 et $22,4^{\circ} \mathrm{C}$, de température moyenne comprise entre 27,28 et $28,6^{\circ} \mathrm{C}$ et de température maximale comprise entre 33,48 et $34,9^{\circ} \mathrm{C}$. Les températures minimale, moyenne et maximale ont également influencées significativement $(\mathrm{P}<0,05)$ le rendement du sorgho. Ainsi, une amélioration du rendement du sorgho avec l'augmentation de température est observée. Les meilleurs rendements ont été enregistrés pour des valeurs de températures minimales allant de 21,90 à $22,4^{\circ} \mathrm{C}$, de températures moyennes allant de 27,21 à $28,6^{\circ} \mathrm{C}$, et de température maximale allant de 33,47 à $34,9^{\circ} \mathrm{C}$.

Tableau 3. Equation de régression entre le rendement des spéculations et les paramètres climatiques dans la Commune de Bembèrèkè

\begin{tabular}{|c|c|c|c|}
\hline Spéculations & $T \min$ & Tmax & Tmoy \\
\hline Maïs & $\begin{array}{ll}\mathrm{AR}=\quad-0,61+0,98 & \mathrm{An} \\
\text { Tmin; } p=0,0000513 & \end{array}$ & NS & $\begin{array}{l}\text { AR= } 0,22+0,95 \quad \text { An } \\
\text { Tmoy ; p=0,007 }\end{array}$ \\
\hline Sorgho & $\begin{array}{ll}\mathrm{AR}= & -0,38-0,64 \quad \mathrm{An} \\
\mathrm{Tmin} ; \mathrm{p}=0,018 & \end{array}$ & $\begin{array}{l}\mathrm{AR}=\quad-0,26+0,59 \quad \mathrm{An} \\
\text { Tmax ; } \\
\mathrm{P}=0,001\end{array}$ & $\begin{array}{l}\mathrm{AR}=\quad 0,34+1,21 \quad \mathrm{An} \\
\text { Tmoy } ; \mathrm{p}=0,0006\end{array}$ \\
\hline Niébé & $\begin{array}{l}\mathrm{AR}=\quad-0,51+0,85 \mathrm{An} \\
\text { Tmin; } \mathrm{p}=0,001\end{array}$ & $\begin{array}{lll}\mathrm{AR}=\quad-0,22+0,48 & \mathrm{An} \\
\mathrm{Tmax} ; & & \\
\mathrm{p}=0,012 & & \\
\end{array}$ & $\begin{array}{l}\mathrm{AR}=-0,32+1,19 \mathrm{An} \\
\text { Tmoy; } \\
\mathrm{p}=0,0008\end{array}$ \\
\hline Arachide & $\begin{array}{l}\mathrm{AR}=\quad-0,44+0,70 \mathrm{An} \\
\text { Tmin; } \mathrm{p}=0,007\end{array}$ & $\mathrm{NS}$ & $\begin{array}{ll}\mathrm{AR}=0,18+0,78 \quad \mathrm{An} \\
\text { Tmoy ; } \\
\mathrm{p}=0,035\end{array}$ \\
\hline
\end{tabular}

\section{Commune de Kandi}

Plusieurs relations significatives ont été établies entre le rendement et les paramètres climatiques de 1981 à 2010 dans la Commune de Kandi (Tableau 4). Les températures maximales expliquent significativement $(\mathrm{P}<0,05)$ le rendement de l'arachide à Kandi. Ainsi, on observe qu'une température maximale comprise entre 34,92 et $35,8^{\circ} \mathrm{C}$ améliore le rendement de l'arachide. En ce qui concerne le maïs, les températures minimales expliquent de manière significative $(\mathrm{P}<0,05)$ les rendements obtenus dans la Commune de Kandi de 1981 à 2010. Mais, le rendement est positivement corrélé avec les 
températures minimales. Ainsi, une température minimale de valeur comprise entre 22,22 et $22,6^{\circ} \mathrm{C}$ améliore le rendement de maïs. La température minimale et la pluviométrie moyenne ont eu également un effet significatif sur le rendement du niébé. Une température minimale comprise entre 22,20 et $22,6^{\circ} \mathrm{C}$ améliore le rendement de niébé. Les températures minimale et moyenne ont significativement influencé le rendement du sorgho dans la Commune de Kandi de 1981 à 2010. Ces rendements sont meilleurs lorsque la température minimale est comprise entre 22,21 et $22,6^{\circ} \mathrm{C}$ et celle moyenne comprise entre 28,61 et $29,12^{\circ} \mathrm{C}$.

Tableau 4. Equation de régression entre le rendement des spéculations et les paramètres climatiques dans la Commune de Kandi

\begin{tabular}{|c|c|c|c|c|}
\hline Spéculations & $T$ min & Tmax & Tmoy & Pmoy \\
\hline Maïs & $\begin{array}{c}\mathrm{AR}=-0,48+0,56 \\
\text { An Tmin; } \\
\mathrm{p}=0,029\end{array}$ & NS & NS & NS \\
\hline Sorgho & $\begin{array}{c}\mathrm{AR}=-0,56+0,66 \\
\text { An Tmin; } \\
\mathrm{p}=0,008\end{array}$ & NS & $\begin{array}{c}\mathrm{AR}=- \\
0,37+0.44 \\
\text { An Tmoy } \\
\mathrm{p}=0,041\end{array}$ & NS \\
\hline Niébé & $\begin{array}{c}\mathrm{AR}=-0,6+0,73 \\
\text { An Tmin; } \\
\text { p=0,003 }\end{array}$ & NS & NS & $\begin{array}{c}\mathrm{AR}= \\
0,001+0,40 \\
\text { An Pmoy ; } \\
\mathrm{p}=0,027\end{array}$ \\
\hline Arachide & NS & $\begin{array}{c}\text { AR }=0,22- \\
0,47 \text { An } \\
\text { Tmax ; } \\
p=0,025\end{array}$ & NS & NS \\
\hline
\end{tabular}

\subsection{Perception paysanne}

\subsubsection{Perception des producteurs agricoles des manifestations de la variabilité climatique}

Les manifestations de la variabilité climatique sont multiples. L'analyse factorielle de correspondances effectuées sur la matrice de données issues des enquêtes de terrain révèle que les deux premiers axes ont contribué à plus de $80 \%$ à la discrimination de l'information contenue dans la matrice. Les chaleurs excessives ont contribué à $64,39 \%$ à la formation de l'axe 1 (tableau 5). L'axe 1 peut être considéré comme l'axe des manifestations liées à la température. Ce sont les pluies précoces qui ont plus contribuées à la formation de l'axe 2 ; il peut être considéré comme l'axe des manifestations pluviométriques. L'inondation et la mauvaise répartition des pluies sont les manifestations les plus citées dans les Communes de Kandi et de Nikki (figure 2) alors que dans les Communes de Bembèrèkè et de Tchaourou c'est la rareté des pluies et les vents violents qui dominent la déclaration des producteurs interrogés. 
Tableau 5. Contribution des manifestations à la formation des axes factoriels

\begin{tabular}{|c|c|c|}
\hline Manifestations & \multicolumn{1}{c}{ Axe 1 } & Axe 2 \\
\hline Pluies précoce & 0,93 & 45,24 \\
\hline Retard dans l'installation des pluies & 3,18 & 14,73 \\
\hline Rareté des pluies & 12,43 & 10,93 \\
\hline Inondation & 13,62 & 5,64 \\
\hline Mauvaise répartition des pluies & 0,42 & 7,90 \\
\hline Vents violents & 1,61 & 15,14 \\
\hline Excès Chaleur & 64,39 & 0,20 \\
\hline Poche de sécheresse & 2,04 & 0,12 \\
\hline Décalage des saisons & 1,38 & 0,10 \\
\hline
\end{tabular}

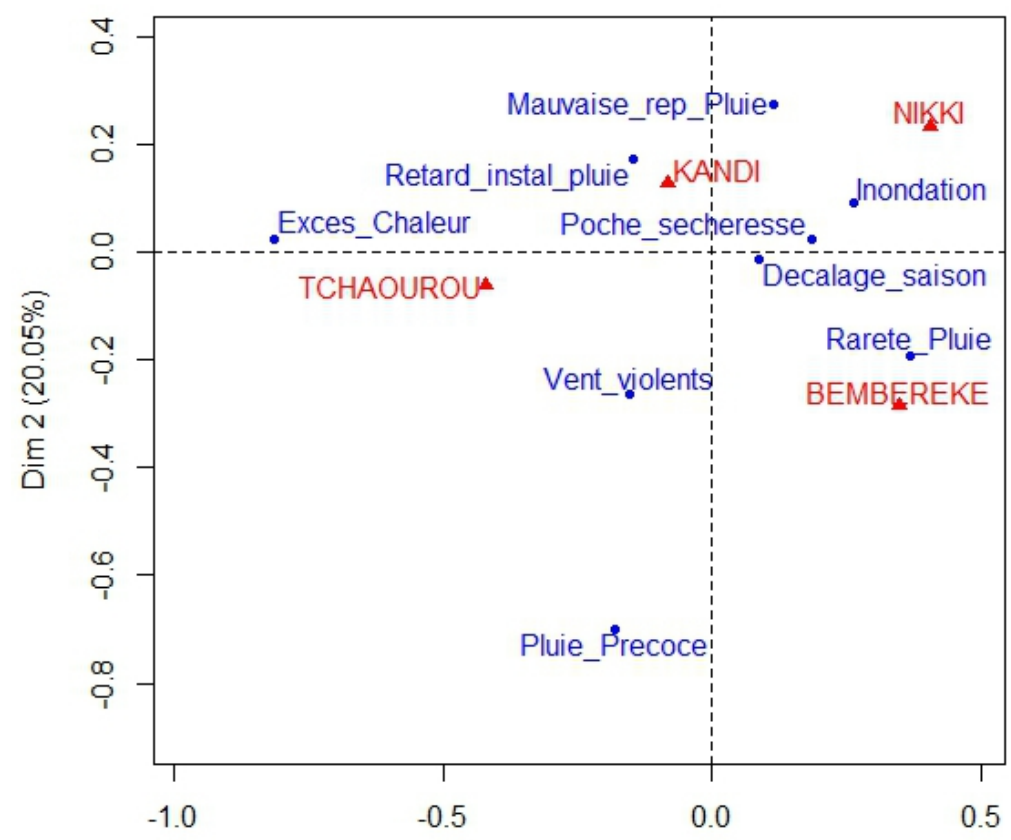

$\operatorname{Dim} 1(65.46 \%)$

Figure 2. Analyse factorielle des correspondances (AFC)

\subsubsection{Causes perçues de la variabilité climatique}

La figure 3 présente les phénomènes perçus par les producteurs agricoles comme causes éventuelles de la variabilité climatique. De l'analyse de cette figure, il ressort que la déforestation (90\% des enquêtés), les phénomènes naturels $(57,92 \%$ des enquêtés), le non-respect des interdits (42,92\% des enquêtés) sont les causes les plus citées par les producteurs 
agricoles. Le test de $\mathrm{Chi}^{2}$ effectué sur les données confirme que la perception des causes de la variabilité climatique ne varie d'une Commune à une autre dans le milieu d'étude $\left(\mathrm{Chi}^{2}=87,739 ; \mathrm{DL}=15\right.$; Valeur de $\left.\mathrm{p}=0,2454\right)$.

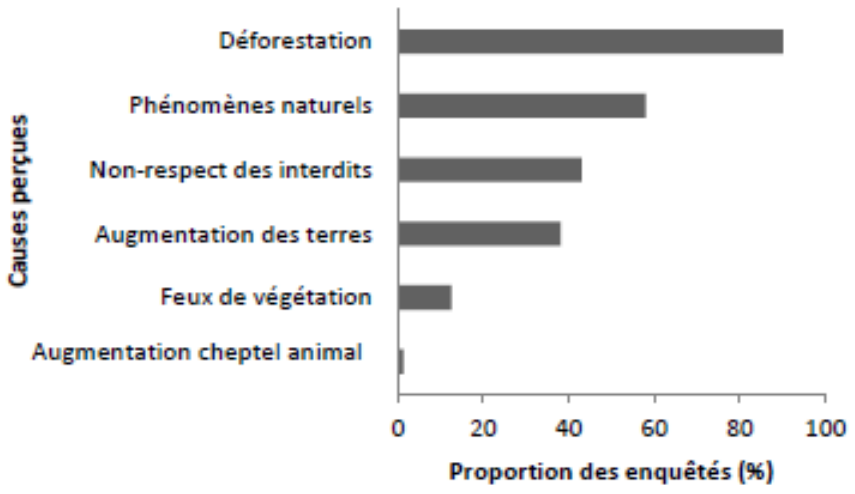

Figure 3. Causes perçues de la variabilité climatique

\subsubsection{Perception des effets des manifestations de la variabilité climatique sur le rendement des cultures vivrières par les producteurs agricoles}

La figure 4 présente l'effet de chaque manifestation de la variabilité climatique sur le rendement. De l'analyse de cette figure, il ressort que toutes les manifestations affectent le rendement agricole (plus $50 \%$ des producteurs affirment une diminution). Aucune des manifestations ne contribue à l'augmentation des rendements mais par contre quelques producteurs reconnaissent que parfois les rendements sont inchangés même en présence des effets de la variabilité climatique.

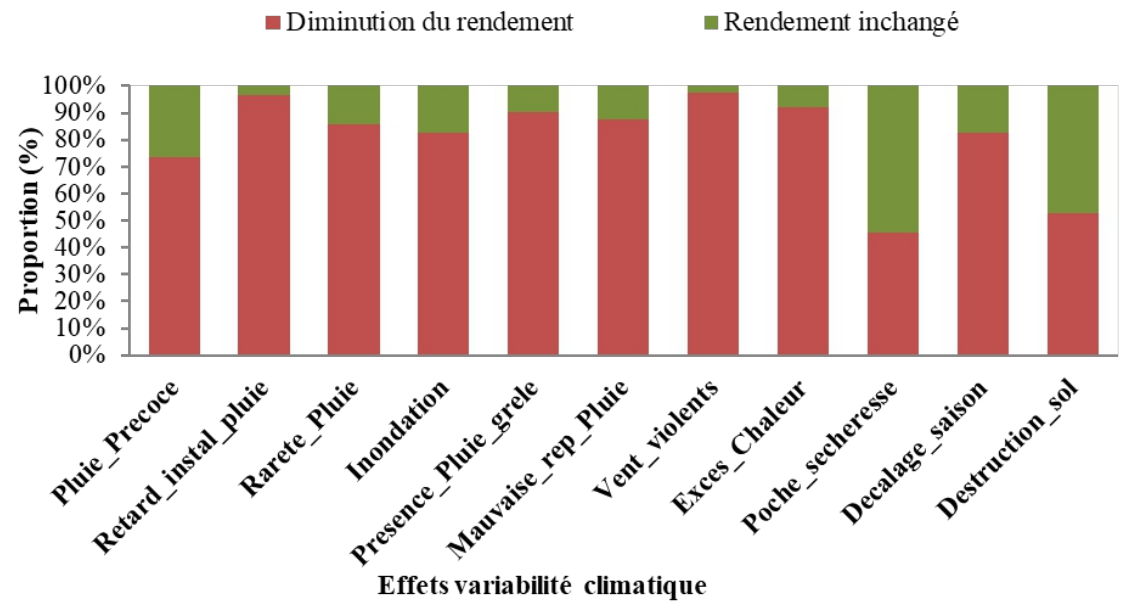

Figure 4. Perception paysanne des effets de la variabilité climatique 


\section{Discussion}

L'analyse de la régression entre les paramètres climatiques et le rendement du maïs, du sorgho, du niébé et de l'arachide a montré que la variabilité des paramètres climatiques a des effets sur les rendements de cultures. En effet, la croissance du maïs varie aussi en fonction des conditions de milieu. Une température inférieure à $15^{\circ} \mathrm{C}$ peut induire un ralentissement de la croissance, affecte donc négativement le rendement (Deffan et al., 2015). Par conséquent, il existe un seuil de température tolérable pour la productivité du maïs. Selon Tidjani et Akponikpe (2012), le maïs est apparu comme la culture la plus vulnérable à la température. Les faibles températures associées à une pluviométrie moyenne améliorent le rendement de maïs. Au regard de l'évolution de ces indicateurs, la production du maïs sera plus hypothéquée les années à venir (Boko et al., 2007; Ogouwalé, 2006). Par contre, le sorgho est une culture adaptée à la zone soudanienne et sahélienne puisqu'il résiste plus à la chaleur (Boyard-Micheau, 2013). Mais malgré cette résistance, l'observation de la pluviométrie et des rendements des cultures pluviales attestent que les années de mauvaise pluviométrie sont globalement suivies d'une baisse des rendements ; le sorgho se révèle très sensible à ces fluctuations (Cabral, 2012). Plusieurs auteurs ont déjà abordé l'effet combiné de la température et de la pluviométrie sur le rendement du sorgho. Avec une température moyenne élevée (environ $35^{\circ} \mathrm{C}$ ) accompagné d'une baisse de pluviométrie engendre de faibles rendements. De plus en plus, les variétés précoces sont privilégiées car ces variétés consomment moins d'eau pendant les premières phases du cycle, ce qui permet un maintien prolongé de la surface foliaire (Amigues et al., 2006). Mais, l'arachide a besoin d'une température de 15 à $45^{\circ} \mathrm{C}$ et un besoin en eau de 400 à $1200 \mathrm{~mm}$, mais la dernière partie du cycle doit être sèche (Ibraheem, 2010). Les déficits hydriques sur une série de processus physiologiques et morphologiques affectent la production de l'arachide par la croissance des pousses et des racines et, finalement, le rendement des gousses (Nigam et al., 2005). L'excès d'eau après la maturation des gousses associé aux fortes températures entraine également des pertes de production liées à la germination sous terre des graines et au développement de Aspergillus flavus L. (un champignon) qui va sécréter l'aflatoxine. La sécheresse est la principale contrainte abiotique qui affecte la productivité et la qualité des arachides dans le monde. Mais un déficit hydrique peut imposer une limitation du rendement (Devi et al., 2009). En culture pluviale, le rendement de l'arachide varie d'une saison à une autre et atteint rarement 1142 tonnes à l'hectare en zones soudano sahéliennes où les températures sont élevées (Thiaw, 2008). Quant au niébé, les analyses de données ont montré que les meilleurs rendements sont obtenus quand la température oscille entre 25 et $28^{\circ} \mathrm{C}$ avec une pluviométrie comprise entre 750 $\mathrm{mm}$ à $1000 \mathrm{~mm}$. Au-delà de cette amplitude, précisément entre 32 et $35^{\circ} \mathrm{C}$, on 
note une chute des fleurs et des gousses (Louni et Tamani, 2015). Ces conditions influencent négativement le rendement de la culture. Le niébé s'adapte bien aux zones tropicales sèches à faible pluviométrie (300-600 mm) et n'est pas très exigeant en sol, mais croît de préférence sur les sols sablolimoneux bien drainés (Dugje et al., 2009). Ainsi, cette variation des paramètres climatiques influe sur la baisse du rendement des spéculations. Ce constat est perçu par les producteurs agricoles ainsi que les manifestations que sont le décalage des saisons, les vents violents, les inondations, les poches de sécheresse, les pluies précoces, le retard dans l'installation des pluies, la rareté des pluies, la mauvaise répartition des pluies (Hounkpè, 2013; Teka et al., 2013; Toffi, 2008). Une concordance existe aussi entre la perception paysanne de l'augmentation des poches de sécheresse et l'évolution croissante des séquences sèches en juillet et en août. On peut donc déduire que les connaissances des populations locales sur l'évolution du climat sont une source importante pour la compréhension scientifique du changement et de la variabilité climatique (Ouoba, 2013). Les variations observées au niveau des paramètres climatiques, surtout la température ambiante et le nombre moyen annuel de jours de pluie, sont également perceptibles au niveau des producteurs agricoles qui arrivent non seulement à confirmer avec beaucoup de certitude les tendances observées, mais aussi à évaluer les conséquences de ce qui en découlent. Au nombre de ces effets, on peut citer les baisses de rendements, les destructions de cultures, le bouleversement du calendrier agricole classique (Houssou-Goe, 2008). La majorité des producteurs sont unanimes sur cette situation constatée. En effet, les perturbations observées ces dernières années ne favorisent plus une bonne production ni un bon rendement. Le climat n'étant pas le seul facteur déterminant le rendement, d'autres facteurs (fertilité du sol, qualité des semences, maladies et ravageurs,...) participent aussi fortement à cette baisse des rendements perçue.

\section{Conclusion}

Dans le nord-est du Bénin, la variabilité des paramètres climatiques que sont la température et la pluviométrie a des effets sur le rendement des cultures. L'analyse de données climatiques et de statistiques agricoles sur la période de 1981 à 2012 a montré que le rendement des cultures de maïs, du niébé, du sorgho et de l'arachide est fortement dépendant de la pluviométrie (Pmoy) et de la température (Tmin, Tmax et Tmoy) dans la zone d'étude. Les faibles températures minimales $\left(21,95\right.$ et $\left.22,4^{\circ} \mathrm{C}\right)$ associées à une pluviométrie moyenne $(1177,20$ et $1490,78 \mathrm{~mm})$ améliorent le rendement de maïs. De même, les meilleurs rendements du sorgho sont obtenus avec la température minimale comprise entre 21,93 et $22,4^{\circ} \mathrm{C}$, moyenne entre 27,31 et $28,6^{\circ} \mathrm{C}$ et la pluviométrie moyenne entre 1136 et $1490 \mathrm{~mm}$. Par contre, pour le niébé et l'arachide, seules les données de températures influencent la qualité des 
rendements. Une température minimale comprise entre 21,90 et $22,6^{\circ} \mathrm{C}$ améliore le rendement de niébé et pour l'arachide, le rendement est meilleur pour une température minimale comprise entre 21,93 et $22,4^{\circ} \mathrm{C}$ et moyenne comprise entre 27,30 et $28,6^{\circ} \mathrm{C}$. Toutefois, au regard de ces résultats, une étude sur la caractérisation des saisons agricoles du milieu s'avère nécessaire pour mieux identifier les périodes de stress pour les cultures, un facteur non négligeable qui influe sur les rendements agricoles.

Les producteurs agricoles, quant à eux, perçoivent les manifestions par le décalage des saisons, les vents violents, les inondations, les poches de sécheresse et les différentes variations au niveau de la pluviométrie. La déforestation, les phénomènes naturels, et le non-respect des interdits sont les phénomènes perçus par les producteurs comme causes éventuelles de la variabilité climatique. Ces perceptions concordent avec les données biophysiques exploitées. Toutefois, l'effet des risques de la variabilité climatique sur le rendement des cultures permettrait de mieux cerner leurs perceptions.

\section{References:}

1. Agbongiarhuoyi, A.E., Abdulkarim, I.F., Fawole, O.P., Obatolu, B.O., Famuyiwa, B.S., Oloyede, A. A. (2013). Analysis of farmers' adaptation strategies to climate change in cocoa production in Kwara State. J. Agric. Ext. 17, pp. 10-22.

2. Agbossou, K.E. (2010). Rapport provisoire d'étude de vulnérabilité, impacts et adaptations de l'agriculture béninoise aux Changements Climatiques. Google Scholar.

3. Amoussou, E., Camberlin, P. et Mahé, G. (2012). Impact de la variabilité climatique et du barrage Nangbéto sur l'hydrologie du système Mono-Couffo (Ouest Afrique). Hydrological Sciences Journal, 57 (4), 805-817

4. Amigues, J.P., Debaeke, P., Itier, B., Lemaire, G., Seguin, B., Tardieu, F., Thomas, A. (2006). Sécheresse et agriculture. Réduire la vulnérabilité de l'agriculture à un risque accru de manque d'eau. Expertise scientifique collective, synthèse du rapport, INRA (France). $72 \mathrm{p}$.

5. Analyse Globale de la Vulnérabilité, de la Sécurité Alimentaire et de la Nutrition (AGVSAN), (2009). Rapport final. http://www.wfp.org/food-security ou wfp.vaminfo@wfp.org, 168p.

6. Ardoin-Bardin, S., 2004. Variabilité hydroclimatique et impacts sur les ressources en eau de grands bassins hydrographiques en zone soudanosahélienne ( $\mathrm{PhD}$ Thesis). Université Montpellier II-Sciences et Techniques du Languedoc. 441 p. 
7. Banque Africaine de Développement (BAD), (2010). Stratégie du secteur de l'agriculture 2010 - 2014. Département de l'agriculture et de l'agro-industrie et département des ressources et politiques opérationnelles, $57 \mathrm{p}$.

8. Boko, M., Niang, I., Nyong, A., Vogel, C., Githeko, A., Medany, M., Osman-Elasha, B., Tabo, R., Yanda, P. (2007). Africa. Climate Change 2007: Impacts, Adaptation and Vulnerability. Contribution of Working Group II to the Fourth Assessment Report of the Intergovernmental Panel on Climate Change, eds. ML Parry, OF Canziani, JP Palutikof, PJ van der Linden and CE Hanson, pp.433-467. Cambridge University Press, Cambridge UK.

9. Boyard-Micheau, J. (2013). Prévisibilité potentielle des variables climatiques à impact agricole en Afrique de l'Est et application au sorgho dans la région du mont Kenya (Doctoral dissertation, Dijon).

10. Cabral, F.J. (2012). Aléas pluviométriques et pauvreté dans les économies du Sahel: le cas du Sénégal, Rainfall fluctuations and poverty in Sahelian economies : evidence from Senegal. Mondes En Dév. pp.129-144.

11. Chede, D.F. (2012). Vulnérabilité et stratégies d'adaptation au changement climatique des paysans du Département des Collines au Bénin : cas de la Commune de Savè (Mastère en changement climatique et développement durable). Centre régional AGRHYMET, Niger, $86 \mathrm{p}$.

12. Dagnelie, P. (1998). Statistique théorique et appliquée vol. 2. Paris, Université De Boeck et Lacier, Paris, 659 p.

13. Deffan, K. P., Akanvou, L., Akanvou, R., Nemlin, G. J., \& Kouamé, P. L. (2015). Evaluation morphologique et nutritionnelle de variétés locales et améliorées de maïs (Zea mays L.) produites en Côte d'Ivoire. Afrique Science: Revue Internationale des Sciences et Technologie, 11(3), 181-196.

14. Devi, MJ, Sinclair, TR, Vadez, V., et Krishnamurthy, L. (2009). Variation génotypique de l'arachide dans l'efficacité de la transpiration et diminution de la transpiration lors du séchage progressif du sol. Field Crops Research , 114 (2), 280-285.

15. Dugje, I.Y., Omoigui, L.O., Ekeleme, F., Kamara, A.Y., Ajeigbe, H. (2009). Production du niébé en Afrique de l'Ouest: Guide du paysan. IITA Ib. Niger.

16. GIEC, E. (2007). Bilan 2007 des changements climatiques. Contribution des Groupes de travail I, II.

17. Honkpehedji, N.R., Agbo, S.A.J. (2009). Analyse des déterminants de la production des cultures vivrières au Benin: cas du maïs et de 
l'igname. (Mémoire d'Ingénieur statisticien économiste). ENEAM/UAC, Bénin.

18. Hounkpè, K. (2013). Impacts des fluctuations climatiques et des activités anthropiques sur les écosystèmes de la plaine de l'Ôti (Togo) (Thèse de Doctorat de Biologie de développement). Lomé, Faculté des sciences. $142 \mathrm{p}$.

19. Hounkponou, K.S., Nouatin, G., Ahounou, M., Atinkpahoun, C.N.H. (2011). Améliorer l'accès des communautés rurales aux informations agro-météorologiques pour renforcer leurs capacités d'adaptation au Bénin: une évolution constructive. Afr. Sociol. Rev. Afr. Sociol. pp.425.

20. Hounzinme, S.S., Teka, O., Oumorou, M. (2017). Stratégies d'adaptation des producteurs agricoles face à la variabilité climatique dans le domaine soudanien au Bénin. Afr. Sci. 1, pp.162-175.

21. Houssou-Goe, S.S.P. (2008). Agriculture et changement climatique au Bénin: risques climatiques, vulnérabilité et stratégies d'adaptation des populations rurales du département du Couffo (Thèse d'Ingénieur Agronome) Faculté des Sciences Agronomiques/Université Nationale du Bénin. 160 p.

22. Ibraheem, A. (2010). L'effet de la température et de la sécheresse sur les variétés locales d'arachide Bambara (Vigna subterranea (L) Verde). Une thèse de doctorat présentée à l'Université de Nothingham .

23. Ikheloa, E.E., Ikpi, A.E., Ikpi, V.O., Oluwatayo, I.B. (2013). Understanding Farmers' Response to Climate Variability in Nigeria: A Multinomial Logit Approach. Ethiop. J. Environ. Stud. Manag. 6, pp.630-369.

24. Issa, M.S. (2012). Changements Climatiques et agrosystèmes dans le Moyen Bénin: impacts et stratégies d'adaptation. Thèse de Doctorat Unique, Université d'Abomey-Calavi, 273p.

25. Louni, H., \& Tamani, F. (2015). Effet bio insecticide de l'huile d'olive et de l'acide oléique à l'égard de Callosobruchus maculatus (Coleoptera-Bruchidae), en utilisant deux substrats: le niébé et le pois chiche (Doctoral dissertation, Université Mouloud Mammeri).

26. Nigam, SN, Chandra, S., Sridevi, KR, Bhukta, M., Reddy, AGS, Rachaputi, NR, ... \& Basu, MS (2005). Efficacité des approches physiologiques et empiriques de sélection pour la tolérance à la sécheresse chez l'arachide. Annales de biologie appliquée, 146 (4), 433-439.

27. Odjugo, P.A., n.d. O (2010). General overview of climate change impacts in Nigeria. J. Hum Ecol 29, pp.47-55. 
28. Ogouwalé, E. (2006). Changements climatiques dans le Bénin méridional et central: indicateurs, scénarios et prospective de la sécurité alimentaire. These de Doctorat unique, LECREDE/FLASH/EDP/UAC.

29. Ouedraogo, N. (2010). Vulnérabilité et pauvreté énergétique, changement climatique et développement socio-économique de l'Afrique sub-saharienne. In Conférence Mondiale sur l'Energie.

30. Ouoba A. P. (2013). Changements climatiques, dynamique de la végétation et perception paysanne dans le Sahel burkinabè (Thèse de doctorat unique). Géographie, Université de Ouagadougou, 305 p.

31. R, CoreTeam (2016). A language and environment for statistical computing. R Foundation for Statistical Computing, Vienna, Austria.

32. Sultan, B. (2011). L'étude des variations et du changement climatique en Afrique de l'Ouest et ses retombées sociétales. Habilit. À Dir. Rech. Univ. Pierre Marie Curie, 137 p.

33. Teka, O., Houessou, G.L., Oumorou, M., Vogt, J., Sinsin, B. (2013). An assessment of climate variation risks on agricultural production: Perceptions and adaptation options in Benin. Int. J. Clim. Change Strateg. Manag. 5, https://doi.org/10.1108/17568691311327578

pp.166-180.

34. Thiaw, C. (2008). Bioactivite des extraits de Calotropis procera AIT. et de Senna occidentalis L. sur Caryedon serratus (ol.), ravageur des stocks et semences d'arachide au Sénégal (Thèse de doctorat de troisieme cycle). FST/UCAD, Sénégal, 182 p.

35. Tidjani, M.A., Akponikpe, P.B.I. (2012). Évaluation des stratégies paysannes d'adaptation aux changements climatiques: cas de la production du maïs au Nord-Bénin. Afr. Crop Sci. J. 20, pp.425-441.

36. Toffi, M. (2008). Le climat, l'homme et la dynamique des écosystèmes dans l'espace littoral du Bénin. Thèse unique de Doctorat Gestion de l'environnement, UAC/FLASH/EDP, Abomey-Calavi, 361 p.

37. Yegbèmey, R.N., Yabi, J.A., Aïhounton, G.B., Paraïso, A. (2014). Modélisation simultanée de la perception et de l'adaptation au changement climatique : cas des producteurs de maïs du nord Bénin (Afrique de l'ouest). Cash Agriexx 1-11p. https://doi.org/10.1684/agar.2014.0697 